\title{
Erratum
}

\section{Simple approximation scheme for the Anderson impurity Hamiltonian}

\section{G. Zwicknagl ${ }^{1}$, V. Zevin ${ }^{2}$, and P. Fulde}

Max-Planck-Institut für Festkörperforschung, Heisenbergstrasse 1, W-7000 Stuttgart 80, Federal Republic of Germany

Z. Phys. B - Condensed Matter 79, 365-375 (1990)

The paper contains the following misprints:

1) p. 367 , Eq. (15) must read:

$\omega \simeq \frac{\Gamma}{\pi} \sum_{m} \ln \frac{\left|\varepsilon_{f m}\right|}{\mathrm{D}+\left|\varepsilon_{f m}\right|}$

2) p. 369 , Fig. 1

The scale on the vertical axis must read $\Gamma / T_{0} \times 10^{-1}$.

The figure caption has to be changed accordingly

"Variation of $\Gamma / T_{0} \times 10^{-1}$ with the low temperature $f$ valence..."

3) p. 372 Lines following Eq. (45)

$\ldots \bar{\omega}=\frac{\omega}{T_{0}} \quad$ and $\quad \bar{w} \ldots$

4) p. 372 , Eq. (47) must read

$$
\chi^{\prime \prime}(\omega, 0)
$$

$=\chi(0) \bar{w}\left\{\frac{\bar{\omega}}{\bar{\omega}^{2}+\bar{w}^{2}} \frac{1}{1+\bar{\omega}}+\frac{2 \bar{\omega}}{\left(\bar{\omega}^{2}+\bar{w}^{2}\right)^{2}} \ln (1+\bar{\omega})\right.$

$$
\left.+\frac{1}{\bar{w}} \operatorname{Im}\left[\left(\frac{1}{\bar{\omega}-\mathrm{i} \bar{w}}\right)^{2} \ln \left(1-\frac{\bar{\omega}}{1+\mathrm{i} \bar{w}}\right)\right]\right\}
$$

5) p. 374, Eq. (A.2) last line must read

$$
\ldots+\frac{1}{\bar{w}} \operatorname{Im} \frac{1}{\left(\bar{\omega}_{m m^{\prime}}-\mathbf{i} \bar{w}\right)^{2}} \ln \left(1-\frac{\bar{\omega}}{1+\bar{\Delta}_{m^{\prime}}+\mathbf{i} \bar{w}}\right) ; \quad \omega \geqq 0
$$

6) Eq. (A.4) first line:

$\ldots a_{\frac{1}{2},-\frac{1}{2}}(\omega, 0) \ldots$

second line:

$\left\langle\ldots\left|J_{x}\right| \ldots\right\rangle$

7) Eq. (B.2) first line

$M_{\alpha}=-\frac{\partial \omega_{0}}{\partial H_{\alpha}}+\ldots$

8) p. 375, Eq. (C.1) must read

$\rho_{f m}(\omega)=\frac{1+\mathrm{e}^{-\beta \omega}}{Z_{f}} \int_{-\infty}^{+\infty} \mathrm{d} \omega^{\prime} \rho_{0}\left(\omega^{\prime}\right) \rho_{m}\left(\omega+\omega^{\prime}\right) \mathrm{e}^{-\beta \omega^{\prime}}$ 